Revue Revue de l'histoire des religions

de Ihistoire des religions

Théologie politique et sciences sociales : autour d'Erik Peterson (1890-1960)

Catholicisme, culture et société aux Temps modernes. Mélanges offerts à Bernard Dompnier. Textes réunis par Cécile DAVY-RIGAUX, Grégory GouDOT, Bernard HOURS et Daniel-Odon HUREL

Turnhout, Brepols («Église, liturgie et société dans l'Europe moderne », 6), 2018

Stefano Simiz

\title{
OpenEdition
}

Journals

Édition électronique

URL : https://journals.openedition.org/rhr/11373

DOI : $10.4000 /$ rhr. 11373

ISSN : 2105-2573

Éditeur

Armand Colin

Édition imprimée

Date de publication : 1 septembre 2021

Pagination : 569-573

ISBN : 978-2-200-93377-7

ISSN : 0035-1423

Référence électronique

Stefano Simiz, «Catholicisme, culture et société aux Temps modernes. Mélanges offerts à Bernard

Dompnier. Textes réunis par Cécile Davy-Rigaux, Grégory Goudot, Bernard Hours et Daniel-Odon Hurel », Revue de l'histoire des religions [En ligne], 3 | 2021, mis en ligne le 01 septembre 2021, consulté le 04 octobre 2021. URL : http://journals.openedition.org/rhr/11373; DOI : https://doi.org/10.4000/rhr. 11373

Ce document a été généré automatiquement le 4 octobre 2021.

Tous droits réservés 


\section{Catholicisme, culture et société aux Temps modernes. Mélanges offerts à Bernard Dompnier. Textes réunis par Cécile DAVY-RIGAUX, Grégory GoudoT, Bernard Hours et Daniel-Odon HUREL}

Turnhout, Brepols («Église, liturgie et société dans l'Europe moderne », 6), 2018

\section{Stefano Simiz}

\section{RÉFÉRENCE}

Catholicisme, culture et société aux Temps modernes. Mélanges offerts à Bernard Dompnier. Textes réunis par Cécile DAvy-RIGAux, Grégory GouDot, Bernard Hours et Daniel-Odon HUREL, Turnhout, Brepols («Église, liturgie et société dans l'Europe moderne », 6), 2018, 346 p., $28 \mathrm{~cm}, 75 €$, ISBN 978-2-503-57953-5.

1 Comment rendre hommage à un collègue qui, par ses champs d'investigation et ses méthodes d'analyse, par sa personnalité aussi, a marqué durablement un secteur important de la recherche historique, en l'occurrence l'histoire religieuse et culturelle des Temps modernes? Pour certains en rassemblant des textes épars en un florilège stimulant; pour d'autres en demandant à des collègues, disciples et/ou amis au sein d'un volume de contributions inédites. Bernard Dompnier (BD) bénéficie des deux attentions. En effet, dès 2013, un ensemble d'articles significatifs, pour la plupart traduits en italien, a été offert aux lecteurs transalpins (I linguaggi della convinzione religiosa. Una storia culturale della Riforma cattolica, Rome, Bulzoni editore, 2013, 267 pages). Cette publication, assez rare il faut en convenir, témoigne du rayonnement scientifique de l'historien français de part et d'autre des Alpes. Deux ans plus tard 
Daniel-Odon Hurel et Bernard Hours ont constitué une autre anthologie donnant un aperçu de son œuvre innovante (Missions, vocations, dévotions. Pour une anthropologie historique du catholicisme moderne, Lyon, Chrétiens et Sociétés, Documents et Mémoires $\mathrm{n}^{\mathrm{o}} 26,2015,445$ pages).

Dernier opus d'une trilogie en hommage à $\mathrm{BD}$, le présent ouvrage est divisé en quatre sections égales de cinq textes chacune et envisage la "complexité du catholicisme moderne ", un "catholicisme pensé et vécu au cœur de la société d'Ancien Régime ». Les auteurs ont tous travaillé et travaillent encore avec BD et représentent bien l'ensemble des domaines qui continuent à passionner l'historien. Pour la commodité et la clarté du propos, les thèmes d'études sont distingués en quatre temps, sur lesquels nous allons revenir, mais le lecteur aurait tort de les considérer comme séparés ou étanches tant c'est la même ambition totale et sociale de compréhension du christianisme catholique tridentin qui transpire à chaque page : réguliers, dévotions, cérémonies, confréries, liturgies, saintetés, traditions, tous ces mots-clés des travaux de BD se recoupent. Signe du fort tropisme souvent nécessaire au chercheur sur la catholicité, parmi les 20 auteurs on compte 14 Français et 6 Italiens. Pour concrétiser un tel projet il faut savoir s'accorder du temps. Commencé en 2014 il a fallu quatre années pour en voir l'aboutissement, période au cours de laquelle deux des contributeurs nous ont quittés : Paola Vismara en 2015 et Xavier Bisaro en 2018. Sans le vouloir, ce livre contient comme un hommage dans l'hommage.

C'est naturellement aux "réguliers dans le cloître et dans le monde ", secteur de recherche par lequel BD s'est d'abord fait connaître, qu'est consacrée la première section du livre. Grâce à Frédéric Meyer, on découvre l'itinéraire singulier de Germain Allart, ce récollet $d u \mathrm{XVII}^{\mathrm{e}}$ siècle qui a tant fait pour l'essor de son jeune ordre et notamment sa réimplantation au Canada, passant «du froc à la mitre » en devenant évêque de Vence. L'historien chambérien a malicieusement choisi une figure illustrant son propre passage des réguliers aux prélats ! Marie Viallon s'interroge pour sa part sur la délicate gestion au sein des servites du cas Paolo Sarpi, par lequel le scandale entre Venise et Rome est survenu en 1606. Dans « Du rififi au couvent », elle propose l'analyse d'une source contemporaine très peu connue et qui rapporte une intrigue «à la Dumas » avec une tentative d'empoisonnement du frère jugé fautif, par ailleurs ancien supérieur des servites. Grégory Goudot prend à bras-le-corps la question des tensions générationnelles émergeant au cœur d'une famille régulière à l'heure des changements. En l'occurrence, la jeunesse est-elle la cause de la déformation, cette forme de peur ressentie face au changement, ou le moyen de la réformation, actualisation nécessaire du projet conventuel aux besoins du temps? Pour Stefania Nanni, le processus de réflexion qui conduit entre 1582 et 1604 les carmes déchaux à choisir « l'option apostolique » et un zèle missionnaire au sein d'une tradition plutôt contemplative et "observante ", est un cas d'espèce particulièrement saisissant de la capacité d'adaptation et du potentiel pastoral présent dans les cloîtres. Enfin, Isabelle Brian montre que l'historien peut passer au-delà de "l'image de vitrail» d'une réputation de régularité entretenue par l'ordre même. Par leurs lettres envoyées au supérieur général de la congrégation à la fin des années 1660, les « Messieurs de SainteGeneviève " illustrent à merveille la diversité et les tensions internes, inhérentes à toute famille régulière, révélant un visage moins consensuel. À l'unité de façade des discours répond une appréciation au vrai de la diversité conventuelle et de la pluralité des opinions. 
La seconde section de l'ouvrage reste focalisée sur les « ordres religieux, fenêtre sur la liturgie et les dévotions ", même si sa partie finale ouvre largement sur le chantier musical. Ludovic Viallet s'interroge sur les enjeux de la pratique du chant des heures comme exercice spirituel au sein de l'ordre franciscain. L'analyse comparée de diverses constitutions $\mathrm{du} \mathrm{xv}^{\mathrm{e}}$ siècle permet d'observer la construction de nouvelles traditions d'offices, forme d'antidote à l'oisiveté ou «démon de midi». Des franciscains il est encore question dans le papier original de Daniel-Odon Hurel, choisissant d'illustrer le rapprochement entre bénédictins et franciscains tracé par le célèbre historien des capucins, Ubald d'Alençon, dans un article de 1926, intégralement reproduit en annexe. L'idée est de faire faire l'éloge de François par Benoît. L'exercice de style est intéressant, favorisant néanmoins les raccourcis les plus évidents (comparer Subiaco et les ermitages du xIII ${ }^{\mathrm{e}}$ siècle) pour fixer (et figer ?) la filiation, un jeu entre histoire et hagiographie. Avec Dominique Deslandres, le propos passe au Nouveau Monde. Sur ces terres lointaines, des nouvelles formes d'apostolat, notamment féminines, semblent en usage, comme le révèle l'aventure de Marie Guyart. En apprenant les langues locales, cette mystique dévote parfait une formation lui permettant une initiative pastorale quasi missionnaire. Rien d'étonnant que son fils, Claude, ait employé pour elle le qualificatif rare de "femme apostolique». Spécialiste des ordres réguliers féminins modernes, Marie-Elisabeth Henneau se concentre sur le riche dossier des célébrations par les visitandines de la béatification de François de Sales. L'idée est de l'organiser depuis Annecy et de demander à l'ensemble des maisons de la répéter (liste et dates fournies). Les religieuses l'anticipent même puisque dès 1661 on planifie ce qui n'est rendu possible que par la cérémonie romaine de 1662. Enfin, Bernard Hours porte son attention sur les passionnants "Couplets» ou chants composés au Carmel de Saint Denis dans les décennies 1770-1780, au temps de Madame Louise. Il ressort de cet ensemble de très belles écritures, formellement soignées, avec la mention des timbres ou airs connus qui permettaient de les fredonner, sans oublier un discours de fond qui s'enracine dans l'apologétique du temps et la défense de l'union ébranlée du trône et de l'autel.

5 Déjà célébré par les auteurs précédents, le thème $n^{\circ} 3$, "Musique et musiciens au service du sacré ", rassemble essentiellement les contributions des musicologues. Tous rappellent combien la rencontre entre $\mathrm{BD}$, spécialiste des dévotions et des liturgies, et des historiens de la musique fut aussi originale que féconde. Le fruit de cette mise en commun méthodologique a été le programme MUSEFREM 1790, lancé en 2001, « [une] aventure scientifique, collective et participative » modèle. Sylvie Granger en a été une des chevilles ouvrières et son papier jette un coup de projecteur sur les organistes de Saint-Maur. Ce portrait de groupe d'une soixantaine de titulaires comprend de nombreuses surprises: ainsi six d'entre eux ont été des femmes - car à l'ombre du sanctuaire tout est possible - et leurs conditions de travail n'ont rien à envier (hélas) à nos CDD et statuts précaires actuels. Xavier Bisaro choisit aussi d'honorer cette recherche collective sur les musiciens d'Église en présentant l'apport de quelques savantes plumes l'ayant précédée, tel le chanoine Clerval (mort en 1918), historien de la maitrise ancienne de Notre-Dame de Chartres. L'inscription d'un projet dans une trame historiographique aide à mieux en apprécier la valeur. Pour Jean Duron, le chantier de la formation au chant des enfants de chœur est une préoccupation méthodologique constante. Comment restituer avec la documentation conservée les modalités et les résultats concrets de cette forme d'apprentissage? Qu'attend l'Église de ces « voix d'anges » et comment les maîtrises travaillent-elles ces voix? Thierry Favier s'empare 
de la figure de Paul-Louis de Mondan, connu pour avoir apporté son soutien au malheureux Le Sueur dans l'affaire qui lui fit perdre la direction musicale de la maitrise de Notre-Dame de Paris en 1787. L'auteur ne s'attarde pas sur cette péripétie, mais entreprend de restituer et de mesurer l'apport musical de l'ancien Toulousain, notamment créateur de quelques remarquables Noëls. Quant à Michela Berti, elle nous ramène à Rome, dans l'église nationale de Saint-Yves-des-Bretons. Le sanctuaire joue un rôle appréciable dans la pastorale, l'animation liturgique, en particulier musicale. L'étude précise et inédite des archives rend compte du souci de professionnalisation et d'italianisation, double gage de qualité, qui accompagne la célébration de la fête tutélaire du patron des avocats. Au-delà de l'évident embellissement, cette cérémonie extraordinaire du catholicisme baroque participe d'une certaine encomiastique française au pays des papes.

Dernière section, la quatrième s'interroge sur les liens entre "Des saints et du sacré ». Jean-Marie Le Gall l'ouvre en questionnant le lien du sacré avec l'État. L'affaire semble historiographiquement parlant entendue, et il suffirait pour cela de remonter notamment à Marc Bloch; pourtant l'objet est plus composite qu'il n'y paraît, et ses effets politiques et cultuels ne se limitent pas au monde monothéiste et chrétien. L'article est un petit tour de force s'évertuant à enraciner le sujet dans diverses traditions, à diverses époques, ceci pour visiter à "nouveaux frais » un domaine important. Sans omettre une salutaire prudence quant à l'usage actuel de "désacralisation ", si souvent dans les bouches ou sous les plumes, alors même que la sacralisation reste un objet mal cerné, parfois fuyant. Avec Sara Cabibbo, c'est un domaine important et familier des recherches de BD qui est abordé, celui des confréries, en particulier les archiconfréries romaines. Celle étudiée dans ces pages est consacrée aux stigmates, observée en pleine activité d'accueil à l'occasion du jubilé général de 1725. «La table du pardon » revisite la tradition conviviale et fondamentale du banquet confraternel. Pour Bernadette Majorana, spécialiste reconnue des arts du spectacle, l'étude du théâtre hagiographique catholique du XvII siècle a le pouvoir d'éclairer aussi les objectifs pastoraux de sanctification et de conversion des fidèles. En re-parcourant les écrits de Campanella (Poétique) ou de Tantillo (Oratione) elle donne à voir les mises en scène non pas comme de simples artifices visuels, mais bien comme des discours moralisateurs dans lesquels l'acteur réel est au moins aussi important que le personnage de fiction qu'il incarne (Christ ou saints). Simona Negruzzo ouvre le dossier du mouvement des couronnements (et d'habillage au passage) des vierges, en particulier ces multiples statues (ou gravures) repérables à compter du $\mathrm{XVII}^{\mathrm{e}}$ siècle. Répandu, l'élan concerne les grandes (Lorette) comme les petites dévotions mariales, locales et plus modestes. La piété qui en résulte, bien encadrée par les ordinaires des diocèses, le clergé et d'autres autorités, a certes des accents antiprotestants, mais elle va bien au-delà. Promouvoir le culte marial, c'est renforcer le christocentrisme. La contribution de Paolo Vismara amplifie cet effet marial, en plein XVIII ${ }^{e}$ siècle. En effet, alors même que Muratori compose son livre sur la nécessité d'une piété bien réglée et que les Lumières semblent vouloir favoriser une foi simplifiée, la dévotion à Marie n'en pâtit pas. Consacrée à la « divine bergère » celle-ci vient enrichir le panel de la ferveur à la Mère de Dieu. Loin d'être accidentelle, anachronique ou limitée, cette nouvelle marque de piété gagne l'ensemble de la péninsule, le monde espagnol et connaît un franc succès en Amérique, là où le catholicisme triomphe. 


\section{AUTEUR}

\section{STEFANO SIMIZ}

Université de Lorraine. 